\title{
Research on the Optimal Resource Allocation Policy based on User Characteristics and Behavior under the Environment of Cloud
}

\author{
Rong Wang \\ Hubei University of Science and Technology, \\ Xianning, Hubei Province,437100 China
}

Abstract. In this paper, we research on the optimal resource allocation policy based on user characteristics and behavior under the environment of cloud. Computing resources and the services are in accordance with the rapid demand to provide to the user to realize through the Internet anytime and calculation mode of easy access to a shared resource pool. The main content of cloud computing for the management of computing is power, storage, platform and services. How to finalize optimal resource allocation policy has significant meaning. Our research deal with this issue well, the numerical simulation illustrate the feasibility of the proposed method.

Keywords: Allocation Policy; Cloud Environment; User Characteristics and Behavior; Optimal.

\section{Introduction}

Cloud computing refers to the super computing model, through the Internet connection consists of distributed processing, parallel processing and grid computing technology or the general commercial implementation of the concept of computer science. Cloud computing capacity as a service for users to buy, users don't have to buy high performance hardware and software development, only need to lease or buy the corresponding devices via the Internet. Computing resources and the services are in accordance with the rapid demand to provide to the user to realize through the Internet anytime and calculation mode of easy access to a shared

\author{
Bin Rao \\ Hubei University of Science and Technology, \\ Xianning, Hubei Province,437100 China \\ ${ }^{*}$ Corresponding Author: Bin Rao
}

resource pool. Computing resources allocation is an important component of cloud computing technology, and its efficiency directly affects the performance of the entire cloud computing environment. Because of cloud computing has many unique properties, making the original resource allocation and scheduling algorithm for grid computing has been unable to work effectively in this environment. With the rapid development of computer technology, more and more high demand for Internet users, the scale of the cloud computing becomes more and more large, user is massive magnitude scale. Therefore, how to meet the needs of users of dynamic change, achieve high efficiency, low cost resources reasonable allocation has become a research focus of cloud computing. The whole cloud computing resource allocation of the key is to choose the reasonable resource allocation algorithm and good resource allocation algorithm not only can improve calculation and transmission speed [1-2].

In cloud computing environment, users will be their personal computers or mobile phones a lot of information on the terminal device and processor resources together, work together. This is a large-scale distributed computing model. The model is determined by the carrier of economic scale and is abstract, virtualization and dynamic variable scale. The main content of cloud computing for the management of computing is power, storage, platform and services. The related content through the Internet, to each according to his need to external users, its significance lies in the capacity as a commodity to circulate on the Internet. A key advantage of 
cloud computing: quickly reduce the hardware cost and improve computing power and storage capacity, the user can at very low cost input for the calculation of high quality, rather than buying expensive hardware devices, reinvestment for frequent maintenance and upgrade. User behavior is in a cloud computing environment and the users have access to resources and invoke the service and the order of the holding time unique characteristics of individual behavior and so on the characteristic of user. The user habit degrees are based on user behavior of individual characteristics and user groups of normal threshold value. Selecting rational resource allocation strategy, can satisfy the dynamic requirements of users, improve the quality of service [3-4]. Now have thousands of cloud resources, and user demand for cloud resources species also have very big difference. Due to user demand for cloud computing resources may change at any time, how to quickly and accurately meet the users' personalized, differentiated demand has become a key point to measure the level of cloud services.

The resources reasonable allocation can improve the efficiency of the entire cloud service market management and social resources of fairness and efficiency. Society as a whole, the user use of each terminal is a cloud computing nodes, each node is the use of cloud computing services and as a resource to provide services, from the broad sense, each terminal is in a state of mutual cooperation, to achieve optimal allocation of resources, therefore, scientific and efficient resource allocation strategy determines the optimization of the allocation of resources of the whole society.

To deal with the challenges, in this paper, we research on the optimal resource allocation policy based on user characteristics and behavior under the environment of cloud. Relative to the grid computing, cloud computing is the extension of grid computing and cloud computing to the constraint conditions of resource allocation, path optimization, such as total cost target demand higher. The allocation of resources under the cloud computing environment is still in a stage of development, the existing relevant theory is not very perfect and the lack of systematic.

\section{The Proposed Policy and Methodology}

The Principles of User Characteristics and Behavior Analysis. Traditional collaborative filtering algorithm based on user is mainly according to the similarity of user ask similar ratings are on the same project, produce neighbor sets, according to the neighbor set to predict target user rating and recommend to the user for a project. With the high-speed development of cloud computing, people's work and life increasingly shift to cloud computing platforms, the number of users of cloud computing platform and its proportion increases rapidly. Cloud storage in order to guarantee the high availability of the data, all data nodes in an open position, each Block has multiple copies, the cloud computing platform for users to access behavior model are studied, according to the characteristics of the user access to data appropriately dormancy or close the corresponding node, achieve the goal of reducing energy consumption has great research value in cloud computing and energy saving. By cloud computing high availability, scalability and other features, in terms of storage, each file is divided into fixed size Block for storage and the each Block has multiple copies, these copies random distribution on a node, each node in the running state.

When the system by a certain state in the process of random moved to another state, in the process of the transfer, there is a transfer probability is called the transition probability. If transition probability and the adjacent two states change, namely the next phase of the state and the present state about only and has nothing to do with the past. In the figure one, we show the systematic structure. 


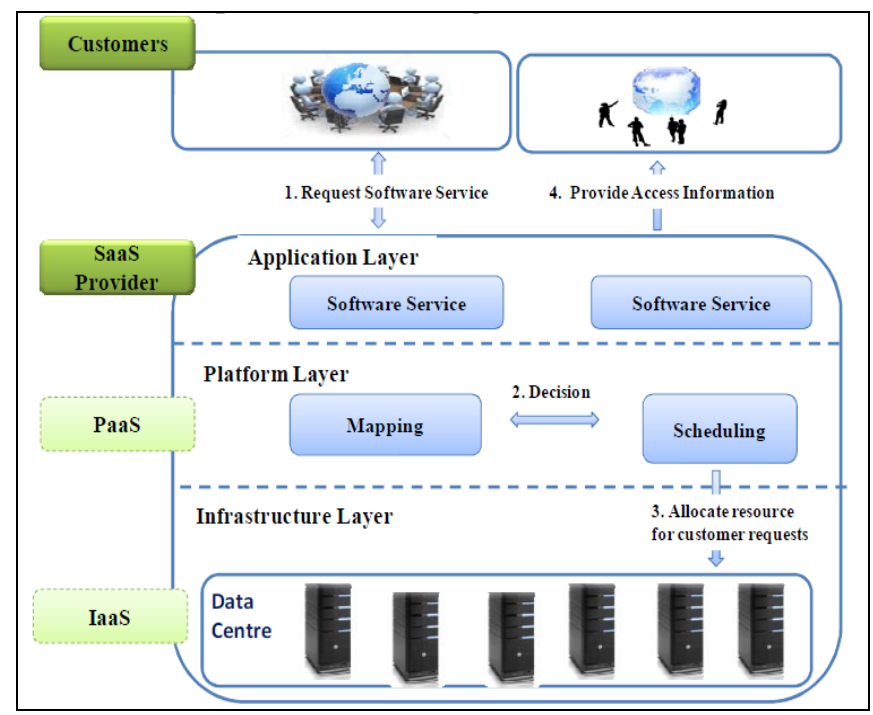

Fig. 1The General Organization of Cloud Environment and User Preference

If the potential for further research and digging, makes servers to find the user access to data characteristics, then according to the user's access features combined with certain algorithm and the strategy, puts some nodes sleep or closed, in terms of improving server utilization, there is a great deal of research value. User program rating matrix sparse sex of similarity measure is not accurate. User project quantity is large, the user program rating data is very thin, if the two may have little or no user similar interests did score for the same project, so similar user is difficult to discern. In the algorithm is recommended when new users join, it is difficult to find a similar system object, cold start problem.
The Optimal Resource Allocation Policy. The algorithms there are multiple tasks in initial resource allocation scheme of competition for resources, the task of reducing the global utility. To this end, the algorithm to achieve the following goals any task of new resource allocation strategy must increase the effectiveness of all tasks. In addition, the algorithm only change once a child task allocation of resources and this change for all other tasks are known. The cloud computing cloud computing resource management by means of the virtual machine, the virtual machine is a special kind of software, can fully simulate the hardware implementation, thus can run on the operating system, and then keep a set of semantic running environment. This article will put forward resource allocation strategy based on user behavior characteristics, the strategy by considering the user work habits and expectations change rule in time, predict a specific time period of cloud computing tasks load distribution and user goals, dynamic adjustment of cloud computing system resources allocation strategy, on the premise of meet the user's expected task is not to maximize and improve overall utilization rate of resources, realize resources to maximize the contribution rate of customer satisfaction. In this way, the execution environment can be transmitted to other physical nodes by means of packaging, isolates the execution environment and physical environment, facilitate the entire application deployment which is shown in the figure two. 


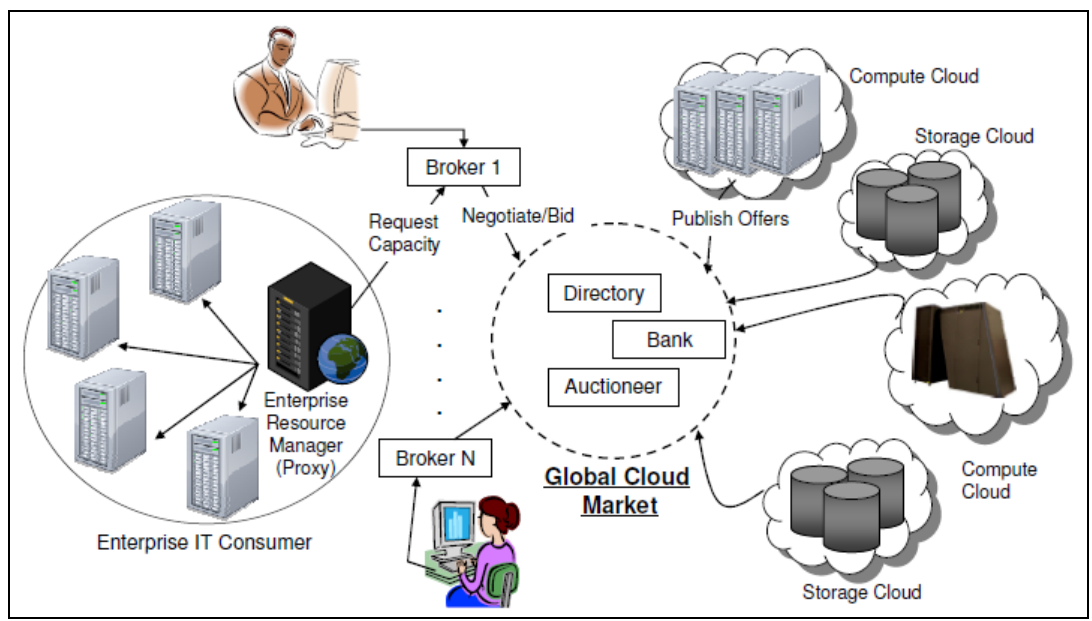

Fig. 2The Deployment of the Resource Allocation

Cloud computing resource scheduling refers to in a given cloud environment, according to the rules of certain resource use, between the different resource user resource scheduling process. At present most of the resource scheduling strategy is through the virtual machine level scheduling technology combined with certain scheduling strategy to do resource scheduling application for the inside of a virtual machine, and the scheduling algorithm is too simple, reduce the performance of the algorithm, so the virtual machine level what algorithm was adopted to realize resource scheduling is a difficult problem to be solved. Cloud resources providers to provide services to consumers, while both to maximize resource utilization, and satisfying the needs of users, but also balance the load, the characteristics of energy consumption and tasks, and cloud computing resource allocation and scheduling problem is a multi-objective optimization problem.

The Optimal Policy under the Environment of Cloud. Resources dynamic allocation strategy for cloud computing environment, the researchers conducted a respectively from different angles of theory and practice. Launches the research from the Angle of resource reliability, considering the resource failure regularity in time and space distribution characteristics, and to rule for the allocation of resources, effective shielding failure of a large number of resource nodes, but this strategy did not consider resource consumption minimization problem, namely the allocated resources of nodes may give a resource demand very small tasks, effective resource utilization is low. Virtual machine the interoperability of cloud computing in a heterogeneous network environment, will be distributed in different structure in the network data resources allocated to different from the network task, need to overcome in the different network data transmission and data protocol of synergy. In a different virtual machine interoperability problems under the network environment will also is need to overcome the technical difficulties in cloud computing. And across data centers to interact with the enormous consumption is on a different platform for cloud computing resource scheduling problem of facing and solving problems. The cloud computing are now a private cloud, cloud computing is public computing network development. While in public computing network for data transmission and the allocation of resources, how to ensure the transmission of data security in public computing in the private cloud and data transmission scheduling algorithm in cloud computing. In the following figure three, we show the corresponding numerical simulation result. 


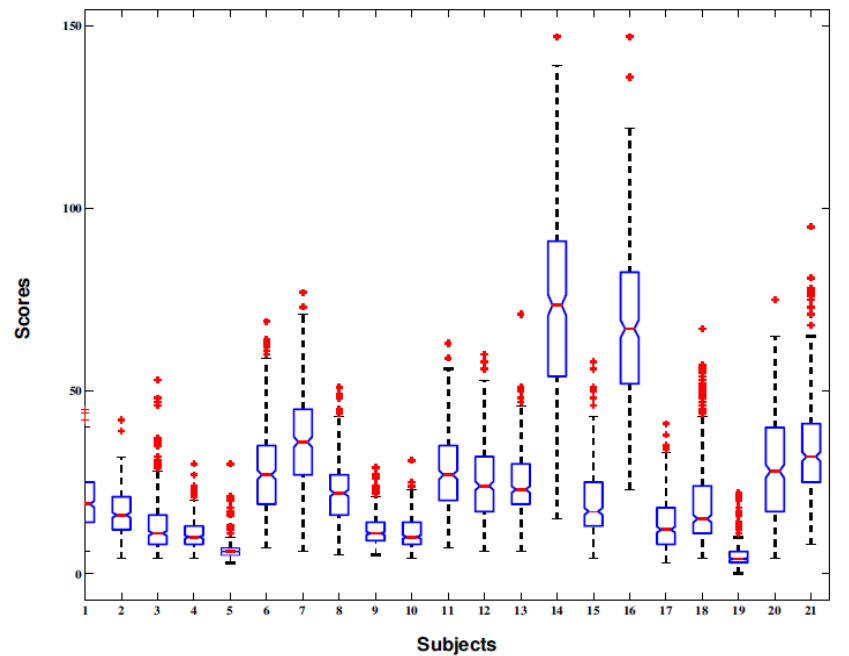

Fig. 3The Simulation and Numerical Analysis of the Optimal Policy

\section{Conclusion}

In this paper, we research on the optimal resource allocation policy based on user characteristics and behavior under the environment of cloud. The current user demand for storage space presents the geometric growth, cloud storage design principle goes against the goal of low carbon energy saving, the excess supply of resources, the results server utilization is low, the serious energy waste problem. The current characteristics of the user to access the study also is less, this article mainly from the study of user data access features, is converted into heat for file access, according to the file access heat dormancy and shut down unnecessary node, in does not affect the service quality and reduce energy consumption so as to realize the purpose of optimal allocation of resources.

\section{Acknowledgement}

The scientific research project of Hubei science and Technology Institute,Number: ky14055

\section{References}

[1] Hoseinyfarahabady M R, Lee Y C, Zomaya A Y. Randomized approximation scheme for resource allocation in hybrid-cloud environment $[\mathrm{J}]$. Journal of Supercomputing, 2014, 69.

[2] Morshedlou H, Meybodi M R. Decreasing Impact of SLA Violations:A Proactive Resource Allocation Approachfor Cloud Computing Environments[J]. Cloud Computing IEEE Transactions.

[3] Bastani P, Mehralian G, Dinarvand R. Resource allocation and purchasing arrangements to improve accessibility of medicines: Evidence from Iran.[J]. Journal of Pharmacy Practice.

[4] Sun J J, Wang X W, Gao C X, et al. Resource Allocation Scheme Based on Neural Network and Group Search Optimization in Cloud Environment[J]. Journal of Software, 2014. 